\title{
Avaliação do uso do agregado reciclado da construção civil como barreira hidráulica em camadas de coberturas de aterros sanitários
}

Evaluation of the use of recycled aggregate from civil construction as a hydraulic barrier in layers of landfill roofs

Evaluación del uso de áridos reciclados de construcción civil como barrera hidráulica en capas de cubiertas de vertederos

Recebido: 06/08/2021 | Revisado: 11/08/2021 | Aceito: 16/08/2021 | Publicado: 18/08/2021

Elivelthon Carlos do Nascimento ORCID: https://orcid.org/0000-0001-6142-6230 Universidade de Pernambuco, Brasil

E-mail:elivelthonnascimento@gmail.com

Kalinny Patricia Vaz Lafayette ORCID: https://orcid.org/0000-0002-7954-2317 Universidade de Pernambuco, Brasil E-mail: klafayette@poli.br

Luciana Cássia Lima da Silva

ORCID: https://orcid.org/0000-0001-6929-9686 Universidade de Pernambuco, Brasil

E-mail: lucianacassialima_@hotmail.com

Jonas da Silva Bezerra

ORCID: https://orcid.org/0000-0002-3880-7614

Universidade Federal de Pernambuco, Brasil E-mail: jonas.silva.doc@gmail.com

Maria Fernanda de Almeida Portela

ORCID: https://orcid.org/0000-0003-2027-4159 Universidade de Pernambuco, Brasil E-mail: mfap_pec@poli.br

\begin{abstract}
Resumo
As camadas de cobertura de aterro sanitário têm a função de impedir a entrada de líquidos bem como evitar a saída de gases. Geralmente são construídas com solos argilosos compactados e de baixa condutividade hidráulica. Neste sentido, esta pesquisa tem como objetivo avaliar a viabilidade do uso de misturas resíduos da construção e demolição (RCD) com solo, para ser utilizado como barreira hidráulica em cobertura final de aterros sanitários. Para isso, foi utilizado um solo argiloso de uma antiga área de extração de saibro desativada a mais de 30 anos, em uma encosta localizada na Ilha de Itamaracá-PE, Região Metropolitana do Recife (RMR). As misturas utilizadas foram de 10\%, $30 \%$ e $50 \%$ de solo e RCD. O programa de investigação contou com ensaios de caracterização física, mecânica e mineralógica, além de ensaios de erodibilidade. Nos ensaios de caracterização física, o solo foi classificado com uma argila de alta plasticidade e o RCD como um material arenoso, cuja adição ao solo provocou uma diminuição do teor de finos do solo até chegar numa argila medianamente plástica, resultados esses, confirmados nos ensaios de Limites de Atterberg. As condutividades hidráulicas saturadas dos solos e das misturas foram da ordem de $10^{-9} \mathrm{~m} / \mathrm{s}$. Os parâmetros de resistência à compressão simples das misturas foram superiores à resistência máxima do solo, tendo alcançado o seu maior valor na mistura de 30\%. Quanto à erodibilidade os ensaios de crumb test e pinhole test indicaram que o solo estudado não apresenta tendência erodível. O acréscimo de RCD diminui a expansibilidade do solo. Desta forma, conclui-se que mistura deste solo específico da região com o RCD é viável para utilização como barreira hidráulica, sendo a mistura de $50 \%$ a que apresentou os melhores resultados.
\end{abstract}

Palavras-chave: RCD; Aterros sanitários; Camadas de cobertura.

\begin{abstract}
The layers of landfill cover have the function of preventing the entry of liquids as well as preventing the escape of gases. They are usually built with compacted clay soils and have low hydraulic conductivity. In this sense, this research aims to evaluate the feasibility of using mixtures of construction and demolition waste (RCD) with soil, to be used as a hydraulic barrier in the final coverage of landfills. For this, a clayey soil from an old clay extraction area that was deactivated for more than 30 years was used, on a slope located on the Island of Itamaracá-PE, Metropolitan Region of Recife (RMR). The mixtures used were 10\%, 30\% and 50\% soil and RCD. The research program included physical, mechanical and mineralogical characterization tests, in addition to erodibility tests. In the physical
\end{abstract}


characterization tests, the soil was classified with a clay of high plasticity and the RCD as a sandy material, whose addition to the soil caused a decrease in the fine content of the soil until it reached a medium plastic clay, results which were confirmed in the tests Atterberg Limits. The saturated hydraulic conductivities of the soils and mixtures were in the order of $10^{-9} \mathrm{~m} / \mathrm{s}$. The parameters of resistance to simple compression of the mixtures were higher than the maximum resistance of the soil, having reached its highest value in the mixture of $30 \%$. Regarding erodibility, the crumb test and pinhole test indicated that the studied soil does not present an erodible tendency. The addition of RCD decreases the expandability of the soil. Thus, it is concluded that mixing this specific soil from the region with the RCD is feasible for use as a hydraulic barrier, with the $50 \%$ mixture having the best results.

Keywords: RCD; Landfills; Covering layers.

\section{Resumen}

Las capas de cobertura del relleno sanitario tienen la función de prevenir la entrada de líquidos así como prevenir el escape de gases. Suelen construirse con suelos arcillosos compactados de baja conductividad hidráulica. En este sentido, esta investigación tiene como objetivo evaluar la factibilidad de utilizar mezclas de residuos de construcción y demolición (RCD) con suelo, para ser utilizadas como barrera hidráulica en la cobertura final de los rellenos sanitarios. Para ello se utilizó un suelo arcilloso de una antigua zona de extracción de grava que había sido desactivada durante más de 30 años, en un talud ubicado en la isla de Itamaracá-PE, Región Metropolitana de Recife (RMR). Las mezclas utilizadas fueron 10\%, 30\% y 50\% de suelo y RCD. El programa de investigación incluyó pruebas de caracterización física, mecánica y mineralógica, así como pruebas de erosionabilidad. En las pruebas de caracterización física se clasificó el suelo como arcilla de alta plasticidad y el RCD como material arenoso, cuya adición al suelo provocó una disminución en el contenido de finos en el suelo hasta alcanzar una arcilla moderadamente plástica, estos resultados fueron confirmado en las pruebas de Atterberg Limits. Las conductividades hidráulicas saturadas del suelos y las mezclas fueron del orden de $10^{-9} \mathrm{~m} / \mathrm{s}$. Los parámetros simples de resistencia a la compresión de las mezclas fueron superiores a la resistencia máxima del suelo, alcanzando su valor más alto en la mezcla al 30\%. En cuanto a la erosionabilidad, la prueba de migajas y la prueba de orificios indicaron que el suelo estudiado no presenta una tendencia erosionable. La adición de RCD disminuye la capacidad de expansión del suelo. Así, se concluye que la mezcla de este suelo específico de la región con el RCD es viable para su uso como barrera hidráulica, siendo la mezcla del $50 \%$ la que presenta mejores resultados.

Palabras clave: RCD; Rellenos sanitários; Cubriendo capas.

\section{Introdução}

A indústria da construção civil é reconhecida como uma das mais importantes atividades para o desenvolvimento econômico e social de um país, apesar disso, ela é uma das grandes responsáveis pela geração de resíduos sólidos, pelo significativo percentual de uso dos recursos naturais e pela degradação ao meio ambiente, sendo considerada como uma das mais consumidoras de recursos naturais (Souza e Balduino, 2020; Manoharan et al., 2020; Yazdanbakhsh, 2018; Park e Tucker, 2016).

Segundo (Akhtar \& Sarmah, 2018) foram gerados 3,0 bilhões de toneladas de resíduos da construção civil no mundo, sendo a China o país que mais produz com 1,13 bilhões de toneladas. No Brasil, por sua vez, são gerados mais de 70 milhões de toneladas por ano e em muitas cidades ainda é comum o descarte irregular. No Estado de Pernambuco, por exemplo, (Paz et al., 2020) georreferenciaram 1252 pontos de descarte de RCD nesta situação, sendo cerca de 80\% constituído por agregados recicláveis, Classes A e B conforme Resolução CONAMA nº 307.

Em virtude dos impactos ambientais causados pelos RCD e da potencialidade de uso como agregado reciclado, diversas pesquisas como (Locastro \& Angelis, 2016; Rios, 2016; Costa, 2015; Dias, 2014) analisaram a viabilidade do uso do agregado reciclado como material alternativo ou complementar, em diferentes proporções em camadas de cobertura de aterros sanitários de Resíduos Sólidos Urbanos (RSU).

As camadas de cobertura finais de aterros de resíduos sólidos urbanos tem como função a separação física entre o resíduo e o meio ambiente, visando à proteção da saúde pública e que, segundo (Santa et al., 2020), faz o isolamento dos contaminantes provenientes dos RSU e o ambienteao redor, além de minimizar a lixiviação e a liberação de gases (Alexandre et al., 2020). Para isso, é fundamental que tais camadas apresentem baixa permeabilidade (Costa et al., 2020). 
(Carvalho et al., 2015) descrevem que é prática comum no Brasil que a impermeabilização da cobertura de aterros sanitários seja executada em camadas de solo compactado na umidade ótima, principalmente com solos argilosos, uma vez que a principal preocupação é promover uma cobertura com baixa permeabilidade ao fluxo descendente de água pela camada, evitando o acréscimo de umidade à massa de resíduos e o fluxo ascendente de gases para a atmosfera.

Especificamente sobre o tipo de solo que deve ser utilizado para cumprir o objetivo de funcionar como barreira hidráulica, a normabrasileira NBR 13896 (ABNT, 1997), menciona apenas a condutividade hidráulica da ordem de $10^{-8} \mathrm{~m} / \mathrm{s}$ como parâmetro geotécnico a ser alcançado. A United States Environmental ProtectionAgency - (USEPA, 2004), por sua vez, define que os solos utilizados para cobertura de aterros sanitários devem ser ricos em argilas e possuir um percentual de finos variando de $30 \%$ a $50 \%$, uma condutividade hidráulica da ordem de $10^{-9} \mathrm{~m} / \mathrm{s}$ e um índice de plasticidade entre $7 \%$ e $15 \%$, além de uma espessura mínima de $0,30 \mathrm{~m}$ e máxima de $0,60 \mathrm{~m}$.

Em virtude disso, este artigo tem como objetivo avaliar as propriedades do solo, agregado reciclado e suas misturas para serem utilizados como barreira hidráulica em um sistema de cobertura final de aterros sanitários.

\section{Metodologia}

Esta pesquisa foi elaborada em caráter exploratório, por tratar uma questão ainda pouco discutida no âmbito da construção civil. Nesse sentido, é necessário aprimorar idéias e descobrir intuições. Desta maneira, foi adotado os métodos quantitativos com base em valores obtidos em ensaios específicos, e com dados numéricos, que visam esclarecer a importância da utilização de RCC em obras de engenharia.

Estes métodos geram conjuntos ou massas de dados que podem ser analisados por meio de técnicas matemáticas, como é o caso das porcentagens, estatísticas e probabilidades, métodos numéricos, métodos analíticos e geração de equações e/ou fórmulas matemáticas aplicáveis a algum processo, (Pereira et al., 2018).

A utilização desta técnica torna uma pesquisa mais forte por compilar informações, acerca dos procedimentos de execução de serviços, em sistema de camada de cobertura de aterros.

A coleta de solo foi uma antiga área de extração de saibro desativada a mais de 30 anos e localizada no Litoral Norte de Pernambuco na Ilha de Itamaracá às margens da PE-035, nas coordenadas $7^{\circ} 46^{\prime}$ 9' $\mathrm{S}$ e $34^{\circ} 50^{\prime} 28^{\prime \prime} \mathrm{W}$, e ocupa uma área de $66,684 \mathrm{~km}^{2}$. A cidade está localizada a $46 \mathrm{~km}$ da capital e possui uma população estimada de 26.672 habitantes e uma densidade demográfica de 328,17 hab $/ \mathrm{km}^{2}$ (IBGE, 2018).

A encosta, que tem em torno de 14 metros, passou por um retaludamento, onde foram inseridas duas bermas, além de um sistema de drenos constituídos de canaletas e escadas d'água para aumentar sua estabilidade. Durante uma inspeção preliminar na encosta foi observado diferenças significativas quanto ao tipo de solo ali presente. Diante disto, foram realizadas coletas em nove pontos, devido a heterogeneidade do material (Figura 1).

Com isso, foi realizado o ensaio de granulometria em cada ponto da coleta conforme a D6913M-17 (ASTM, 2017a). De acordo com a (USEPA, 2004) é indicado a utilização de um solo argiloso para um sistema de cobertura de aterros sanitários. Desta forma foram coletados aproximadamente $250 \mathrm{~kg}$ de amostra deformada do ponto 9, por atender tais condições. Já os agregados reciclados foram coletados na empresa Ciclo Ambiental, uma empresa de beneficiamento dos resíduos da construção civil localizada na cidade de Camaragibe-PE e que recebe os RCD provenientes da Região Metropolitana de Recife. 
Figura 1-Encosta da coleta de solo.

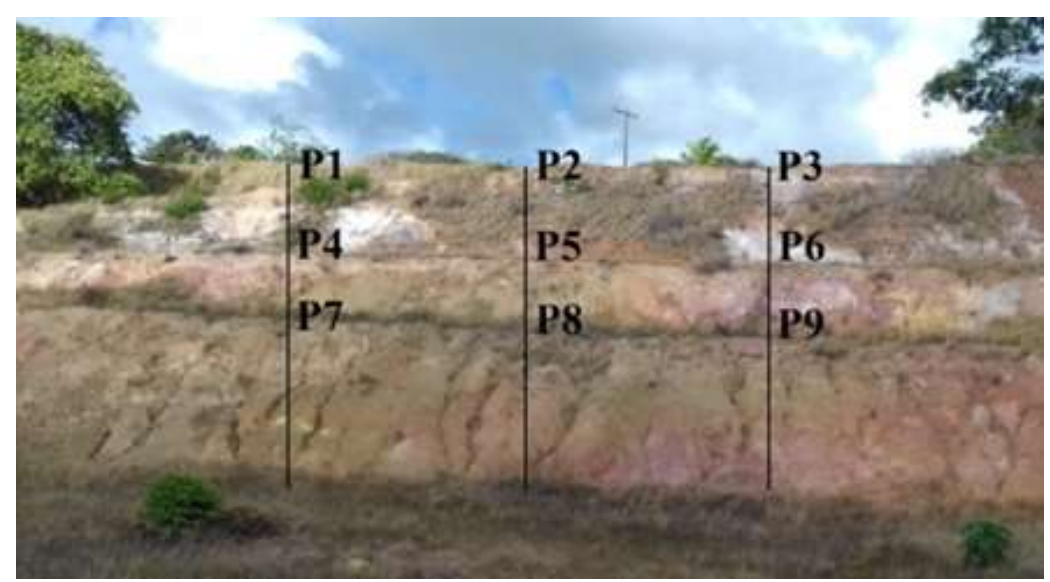

Fonte: Autores.

De posse dos materiais foi definido as misturas de solo-RCD em 10\%, 30\% e 50\% da massa seca do solo, tais misturas receberam a nomenclatura de S90R10, S70R30 e S50R50, respectivamente.

Foram realizados ensaios de caracterização física e mecânica no solo, RCD e nas misturas, além dos ensaios de caracterização mineralógica no solo e no RCD. Os ensaios realizados bem como as normas utilizadas são apresentados na Tabela 1.

Vale destacar que no ensaio de difração de raios x foi utilizado o difratômetro da marca Shimadzu (XRD-6000), com radiação K-alfa do cobre, sendo o pó natural não orientado a forma de análise e com as seguintes condições do equipamento: voltagem de $40 \mathrm{kV}$, amperagem de $30 \mathrm{~mA}$, intervalo de $5^{\circ}$ a $70^{\circ}$ e velocidade de análise $2 \% \mathrm{~min}$.

Tabela 1-Encosta da coleta de solo.

\begin{tabular}{|c|c|c|}
\hline \multicolumn{2}{|l|}{ Ensaios } & \multirow{2}{*}{$\begin{array}{c}\text { Normas } \\
\text { D422-63 (ASTM, 2007) e ASTM D6913M-17 (2017a) }\end{array}$} \\
\hline \multirow[b]{4}{*}{ Caracterização f } & Granulometria & \\
\hline & Densidade real & D854-14 (ASTM, 2014) \\
\hline & Limites de Atterberg & D4318-17E01 (ASTM, 2017b) \\
\hline & Condutividade Hidráulica & NBR 14545 (ABNT, 2000) \\
\hline \multirow{2}{*}{$\begin{array}{l}\text { Caracterização } \\
\text { mecânica }\end{array}$} & Ensaio de compactação & D1557-12 (ASTM, 2012) \\
\hline & $\begin{array}{l}\text { Ensaio de compressão } \\
\text { simples }\end{array}$ & D2166M-16 (ASTM, 2016) \\
\hline \multirow{3}{*}{$\begin{array}{l}\text { Caracterização } \\
\text { mineralógica }\end{array}$} & Lupa & Lupa binocular STEMI 508 com aumento de 50 vezes \\
\hline & Difração por raios-x & Difratômetro da marca Shimadzu (XRD-6000) \\
\hline & $\begin{array}{l}\text { Fluorescência de raios-X } \\
\text { (EDX) }\end{array}$ & $\begin{array}{l}\text { Espectrômetro de fluorescência de raios } x \text { Rigaku } \\
\text { modelo ZSX Primus II, equipado com tubo de } \mathrm{Rh} \text { e } 7 \\
\text { cristais analisadores }\end{array}$ \\
\hline \multirow{3}{*}{ Erodibilidade } & Crumbtest & D6572-13e2 (ASTM, 2013a) \\
\hline & Pinholetest & D4647M-13 (ASTM, 2013b) \\
\hline & Expansibilidade & DNIT $160 / 2012$ \\
\hline
\end{tabular}

Fonte: Autores. 


\section{Resultados e Discussão}

\section{Análise granulométrica}

A análise granulométrica das amostras apresentou o solo como material argiloso com 54,24\%, enquanto o RCD, arenoso com 77,42\%. Como esperado, à medida que o percentual de agregado reciclado incorporado na mistura aumentava, o material se tornou mais granular, com maiores percentuais de areia, conforme mostrado nas curvas granulométricas da Figura 2.

Figura 2- Curva granulométrica do solo, RCD e misturas

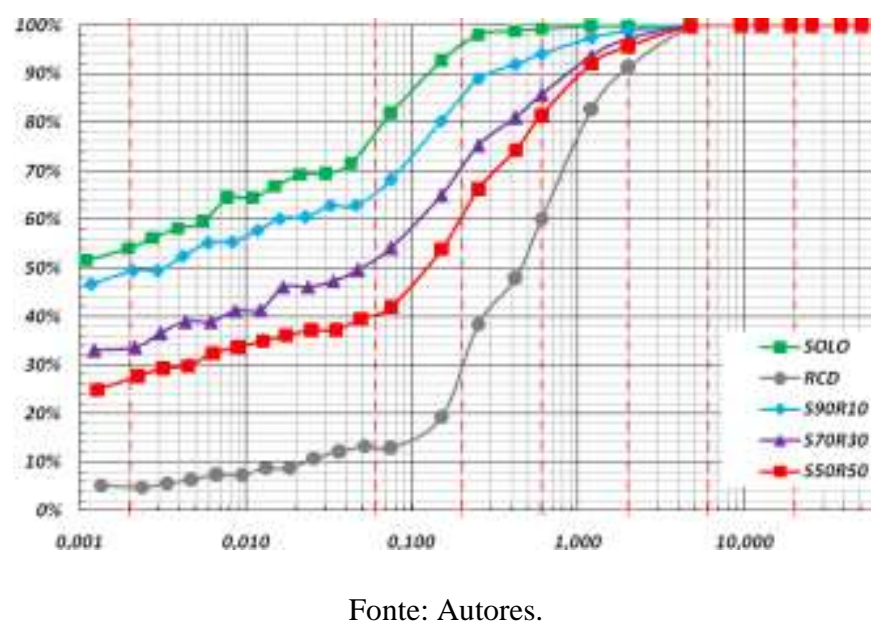

De acordo com o Sistema de Unificado dos Solos (SUCS) o solo foi classificado como uma argila inorgânica de alta plasticidade $(\mathrm{CH})$, as misturas foram classificadas como inorgânica de mediana plasticidade (CL), enquanto que o RCD foi classificado como areia bem graduada (SW).

De acordo com as especificações da Companhia Ambiental do Estado de São Paulo - (CETESB, 1993), o solo e as misturas poderiam ser utilizados como barreira hidráulica. Sendo os resultados encontrados nas misturas semelhantes aos encontrados por autores como (Araújo, 2017; Moraci et al., 2017) estudando solo de aterros experimentais no Brasil e na Itália, respectivamente.

Uma importante análise que relaciona o tamanho dos grãos das partículas ao uso em camadas de aterros como barreira hidráulica é definido pela United States Environmental Protection Agency - (USEPA, 2004), onde o material deve possuir porcentagem de finos variando de 30\% a 50\%. As misturas S70R30 (50\%) e S50R50 (40\%) apresentam tais características.

\section{Densidade Real e Limites de Atterberg}

A Tabela 2 apresenta os valores para densidade real dos grãos, solo, RCD e misturas. Observou-se que o solo foi o material com maior densidade real e o RCD o de menor densidade.

Tabela 2-Encosta da coleta de solo

\begin{tabular}{llllll}
\hline Amostra & Solo & RCD & S90R10 & S70R30 & S50R50 \\
\hline $\begin{array}{l}\text { Densidade } \\
\text { Real } \\
\left(\mathbf{g} / \mathbf{c m}^{3}\right)\end{array}$ & 2,73 & 2,61 & 2,66 & 2,66 & 2,61 \\
\hline
\end{tabular}


Especificamente sobre o RCD, a densidade real foi de $2,61 \mathrm{~g} / \mathrm{cm}^{3}$, tais valores são próximos aos encontrados por

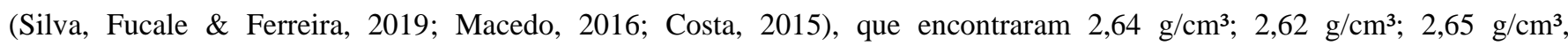
respectivamente. Nota-se ainda que à medida que o percentual de agregado reciclado incorporado na mistura aumentava, a sua densidade real diminuía. Isso se deve ao fato de que o RCD é constituído por grãos maiores aos do solo o que faz com que a sua área específica seja menor e, consequentemente, tenha uma densidade menor.

Os resultados obtidos para os limites de liquidez e plasticidades do solo, RCD e misturas são apresentados na Tabela 3. Nota-se que a adição do RCD nas misturas ocasiona uma diminuição no índice de plasticidade, tal tendência também é observada no trabalho de (Costa, 2015). Vale destacar que com 50\% de RCD no solo o material deixou de ser altamente plástico para medianamente plástico.

Tabela 3 -Limites de Atterberg.

\begin{tabular}{|c|c|c|c|c|}
\hline & $\begin{array}{l}\text { LL } \\
(\%)\end{array}$ & $\begin{array}{l}\text { LP } \\
(\%)\end{array}$ & $\begin{array}{l}\text { IP } \\
(\%)\end{array}$ & Classificação \\
\hline Solo & 53,28 & 21,75 & 31,53 & $\begin{array}{l}\text { Altamente } \\
\text { Plástico }\end{array}$ \\
\hline RCD & NL & NP & - & Não Plástico \\
\hline S90R10 & 42,98 & 20,97 & 22,01 & $\begin{array}{l}\text { Altamente } \\
\text { Plástico }\end{array}$ \\
\hline S70R30 & 36,87 & 20,72 & 16,15 & $\begin{array}{l}\text { Altamente } \\
\text { Plástico }\end{array}$ \\
\hline S50R50 & 31,68 & 20,69 & 10,99 & $\begin{array}{l}\text { Medianamente } \\
\text { Plástico }\end{array}$ \\
\hline
\end{tabular}

Fonte: Autores.

Quanto ao uso como barreira hidráulica em cobertura de aterros sanitários, observando os parâmetros da (USEPA, 2004), tem-se apenas a mistura S50R50 como adequada para uso em aterros devido a sua mediana plasticidade. (Moraci et al., 2017) afirmam que solos altamente plásticos são difíceis de compactar, principalmente em cobertura de aterro. Além disso, são susceptíveis à fissura por retração, nos ciclos de secagem e umedecimento, fazendo com que a condutividade hidráulica seja aumentada.

\section{Condutividade hidráulica}

O solo apresentou a menor condutividade hidráulica, e à medida que o RCD foi incorporado na mistura, a condutividade aumentou, sendo a mistura S50R50 a que apresentou maior variação, cerca de 580\% (Tabela 4). Apesar disso, todas as misturas permaneceram com a condutividade hidráulica na ordem de $10^{-9} \mathrm{~m} / \mathrm{s}$.

Os valores encontrados no solo e misturas possuem uma condutividade hidráulica menor se comparado a valores

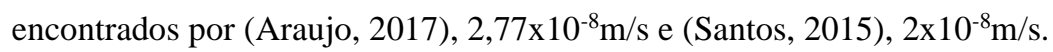

Comparando com os critérios geotécnicos exigidos pela NBR 13896 (ABNT, 1997; USEPA, 2004), para uso como barreira hidráulica em cobertura de aterros sanitários, todos os materiais atendem, sendo a mistura S50R50 a de maior vantagem devido a maior redução de finos e diminuição de plasticidade apresentada. 
Tabela 4-Condutividade hidráulica, à temperatura de $20^{\circ} \mathrm{C}$.

\begin{tabular}{ll}
\hline Material & $\begin{array}{l}\text { Condutividade } \\
(\mathbf{m} / \mathbf{s})\end{array}$ \\
\hline Solo & $1,05 \times 10^{-9}$ \\
RCD & $3,89 \times 10^{-6}$ \\
S90R10 & $1,22 \times 10^{-9}$ \\
S70R30 & $1,77 \times 10^{-9}$ \\
S50R50 & $7,18 \times 10^{-9}$ \\
\hline
\end{tabular}

Fonte: Autores.

\section{Ensaio de compactação}

As curvas de compactação do solo, RCD e misturas são apresentadas na Tabela 5. Nota-se que houve um aumento do peso específico máximo e diminuição da umidade ótima, conforme o acréscimo do teor de resíduo na mistura. Isso pode ser explicado devido ao tamanho das partículas do solo, que está relacionado com a sua área específica, que quanto menor (no caso do solo) precisa de mais água para ser envolvida, e consequentemente apresentará uma menor densidade seca máxima. Ao acrescentar RCD, que tem partículas maiores, a quantidade de água diminui e a densidade seca máxima aumenta.

Tabela 5-Ensaio de compactação.

\begin{tabular}{lcclll}
\hline & Solo & RCD & S90R10 & S30R70 & S50R50 \\
\hline $\begin{array}{l}\text { Umidade } \\
\text { ótima (\%) }\end{array}$ & 28,19 & 12,69 & 21,47 & 16,95 & 16,02 \\
\hline $\begin{array}{l}\text { Densidade } \\
\text { seca } \\
\text { máxima } \\
\left(\mathbf{g} / \mathbf{c m}^{3}\right)\end{array}$ & 1,53 & 1,89 & 1,61 & 1,68 & 1,73 \\
\hline
\end{tabular}

Fonte: Autores.

De acordo com os dados, observa-se que o solo apresenta um peso específico seco máximo de 1,53 $\mathrm{g} / \mathrm{cm}^{3}$ e umidade ótima de 28,19\%, valores próximos ao encontrado por (Costa, 2015), que foi 1,34 g/cm³ para o peso específico máximo e $30,80 \%$ para umidade ótima. Por sua vez, o RCD apresenta um peso específico seco máximo de 1,89 g/cm³ e umidade ótima de $12,69 \%$, valores próximos ao encontrado por (Macedo, 2016) que foi $1,91 \mathrm{~g} / \mathrm{cm}^{3}$ para o peso específico máximo e $13,40 \%$ para umidade ótima.

\section{Ensaio de resistência à compressão simples}

A Figura 3 apresenta a resistência máxima à compressão simples (RSC) nos materiais para 7, 28, 60 e 120 dias, respectivamente. Observa-se que em todos os materiais houve um incremento de aproximadamente $600 \%$ na resistência máxima à compressão, sendo que no agregado reciclado não houve diferenças significativas aos 7 e 28 dias de rompimento. 
Figura 3- Resistência máxima à compressão simples.

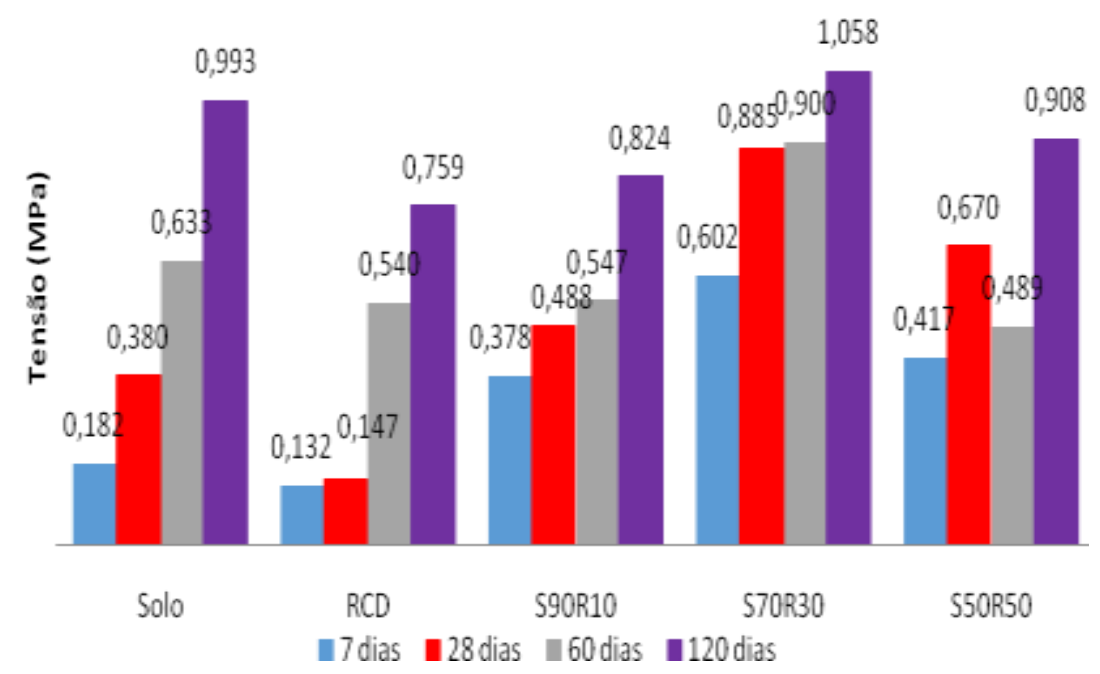

Fonte: Autores.

Nota-se ainda que na adição de RCD ao solo proporcionou um aumento da resistência, tendência observada também na pesquisa de (Costa, 2015). Isso ocorre devido a presença de materiais aglomerantes no resíduo, oriundos das parcelas de argamassas, concretos e materiais cerâmicos.

O solo apresentou um incremento de resistência à compressão de cerca de $450 \%$ entre os rompimentos de 7 e 120 dias, passando de 0,182 MPa para 0,993 MPa. Já o agregado reciclado teve um incremento de $475 \%$ de resistência máxima entre os rompimentos de 7 e 120 dias, passando de 0,123 MPa para 0,759 MPa, tal variação pode ser resultante dos materiais cimentícios presentes no RCD, que variam de acordo com os materiais constituintes.

Nas misturas S90R10, S70R30 e S50R50 houve um incremento na resistência máxima entre os rompimentos de 7 e 120 dias de 118\%; 75\% e 117\%, respectivamente. Nota-se ainda, que foi a mistura S70R30 que apresentou uma melhor resistência, entretanto o aumento foi de apenas $6,5 \%$ se comparado ao solo aos 120 dias.

No que diz respeito ao uso em camadas de cobertura de aterros sanitários, a metodologia de (Daniel \& Wu, 1993) estabelece um valor mínimo de 0,20 MPa, tornando possível, por este método, o uso do solo, agregado reciclado e misturas. Nota-se ainda que o solo só ultrapassa o valor mínimo exigido na metodologia, aos 28 dias, enquanto que o agregado reciclado, aos 60 dias.

\section{Caracterização mineralógica}

$\mathrm{Na}$ análise mineralógica dos grãos do RCD, através da lupa binocular, observou-se a ocorrência de fragmentos de rocha, presença de caulim, biotita, muscovita, além de uma grande quantidade de grão quartzo sendo alguns revertidos por argilominerais. Também foram identificados grãos de quartzo brilhantes e materiais laterizados impregnados de óxidos e hidróxidos de ferro. Em geral, os formatos dos grãos foram de angulosos a subangulosos.

Jáa análise mineralógica por difração de raios-x realizada no RCD, Figura 4, observam-se os picos de caulinita, quartzo e feldspato. A caulinita encontrada é o principal constituinte do mineral caulim que foi encontrado através da lupa binocular, estando presente no RCD indicando a presença de materiais cerâmicos, assim como o feldspato. Já o quartzo é proveniente de material arenoso que pode ser resultante das areias utilizadas no concreto e argamassa presentes no RCD. A análise do solo, por sua vez, indicou a presença de caulinita e quartzo. 
Figura 4- Difratograma de raio X da fração do RCD (a) e do solo (b).
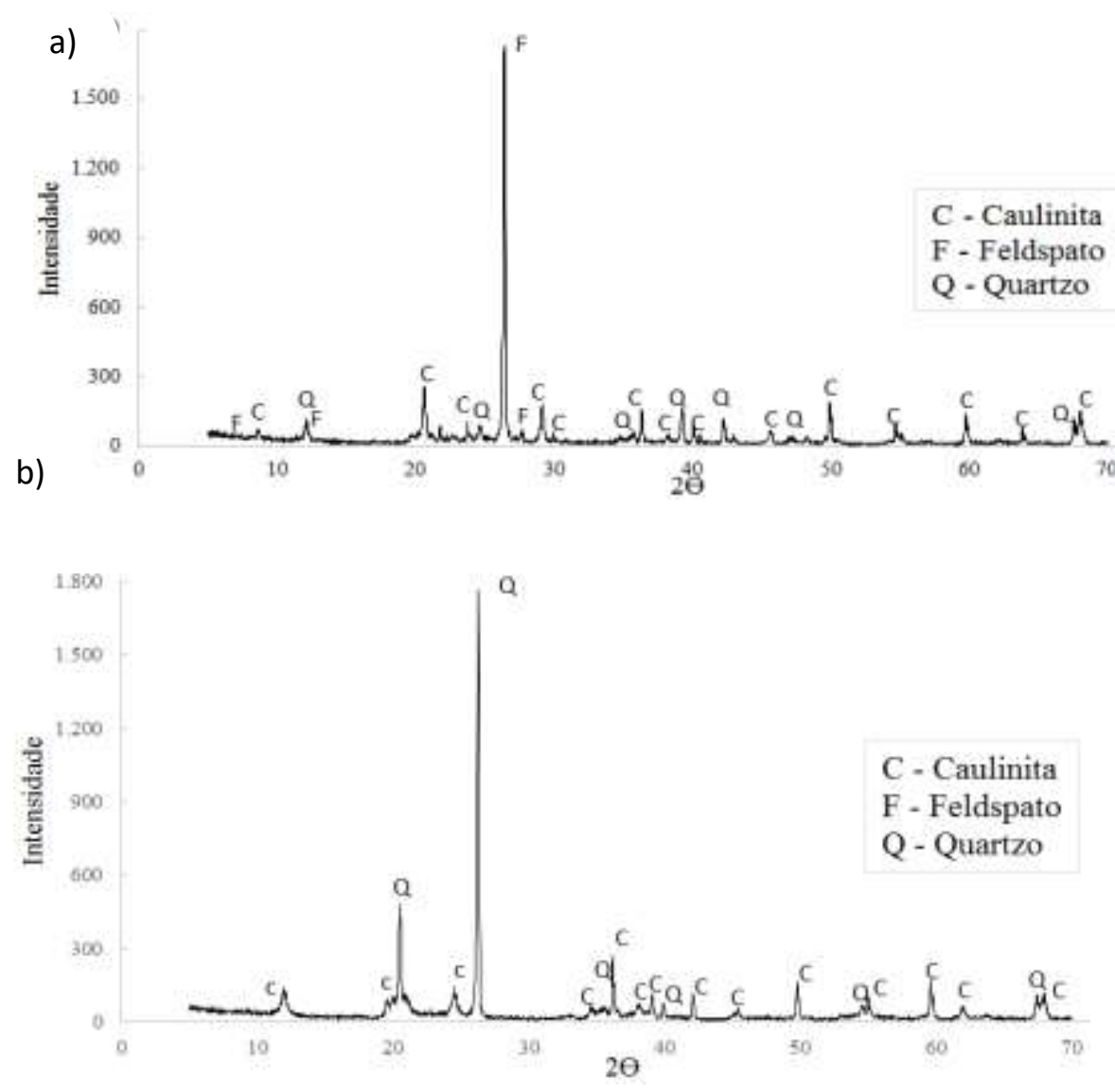

Fonte: Autores.

No RCD o ensaio de fluorescência de raios x mostrou que a sílica (SiO2) é o principal constituinte com 44,01\%, seguido da alumina (A12O3) e do óxido de cálcio $(\mathrm{CaO})$, com teores de 22,02\% e 10,21, respectivamente (Tabela 6). O alto teor de sílica pode estar associado à presença de resíduos de agregados e de argamassa e confirma a presença de areia como demostrado na granulometria e no quartzo que foi encontrado no ensaio de difração de raios x. A indicação de cerâmicas vermelhas é mostrada pelos teores de alumina (A12O3) e óxido de cálcio $(\mathrm{CaO})$. Nota-se ainda que foi encontrado $13,08 \%$ de material que apresentou perda ao fogo (P.F.). 
Tabela 6- Fluorescência de raios do RCD e solo

\begin{tabular}{|c|c|c|}
\hline ELEMENTOS & RCD & SOLO \\
\hline $\mathrm{SiO}_{2}$ & 44,01 & 63,18 \\
\hline $\mathrm{Al}_{2} \mathrm{O}_{3}$ & 22,02 & 20,4 \\
\hline $\mathrm{CaO}$ & 10,21 & 0,01 \\
\hline $\mathrm{Fe}_{2} \mathrm{O}_{3 t}$ & 4,28 & 7,96 \\
\hline $\mathrm{SO}_{3}$ & 2,04 & N. D. \\
\hline $\mathrm{K}_{2} \mathrm{O}$ & 1,57 & N. D. \\
\hline MgO & 1,02 & 0,05 \\
\hline $\mathrm{TiO}_{2}$ & 0,85 & 1,27 \\
\hline $\mathrm{Na}_{2} \mathrm{O}$ & 0,31 & 0,06 \\
\hline $\mathrm{P}_{2} \mathrm{O}_{5}$ & 0,31 & N. D. \\
\hline P.F & 13,08 & 7,07 \\
\hline
\end{tabular}

Fonte: Autores.

Para o solo argiloso o ensaio de fluorescência de raios x mostrou a predominância do óxido de sílica $\left(\mathrm{SiO}_{2}\right)$, óxido de alumínio $\left(\mathrm{Al}_{2} \mathrm{O}_{3}\right)$ e óxido de ferro $\left(\mathrm{Fe}_{2} \mathrm{O}_{3 \mathrm{t}}\right)$. (Ortega \& Campos, 2018) esses óxidos podem estar associados a presença predominante de caulinita, a mesma que é responsável pelo desenvolvimento de plasticidade expansão da argila.

A perda ao fogo (P.F) foi inferior ao RCD, sendo de 7,07\%, essa massa que se perde é resultado, segundo Scarin e Bernardes (2016), da quantidade de argilominerais e hidróxidos de ferro e alumínio presentes no solo, sendo esses produtos do intemperismo. Nota-se ainda que não foram detectados (N. D.) óxidos de enxofre $\left(\mathrm{SO}_{3}\right)$, óxido de potássio $\left(\mathrm{K}_{2} \mathrm{O}\right)$ e pentóxido de fósforo $\left(\mathrm{P}_{2} \mathrm{O}_{5}\right)$.

\section{Ensaios de Erodibilidade}

\section{Ensaio Crumb Test}

Não ocorreu esparramento do solo no fundo do béquer, continuando com seu formato inicial, sendo classificado de Grau 1, ou seja, comportamento não dispersivo. Esse resultado, figura 5, é satisfatório para o uso em cobertura final de aterros sanitários, visto que é um indicador de erodibilidade do solo, característica a ser evitada em cobertura de aterros sanitários. 
Research, Society and Development, v. 10, n. 10, e547101019158, 2021

(CC BY 4.0) | ISSN 2525-3409 | DOI: http://dx.doi.org/10.33448/rsd-v10i10.19158

Figura 5- Ensaio de Crumb Test.

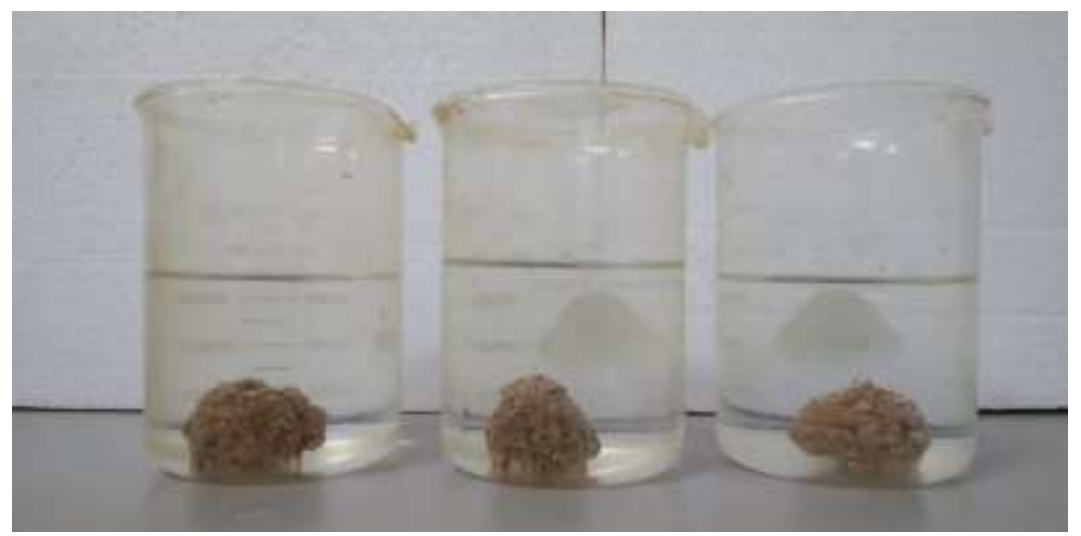

Fonte: Autores.

\section{Ensaio pinhole test}

A Figura 6 apresenta os dispositivos para realização do teste. Já na figura 7 será apresentado o resultado do ensaio de pinhole test.

Figura 6- Ensaio de pinhole: a) tanque para fornecimento de água com carga constante; b) cilindro metálico com diâmetro interno de $35,7 \mathrm{~mm}$ e altura de $100 \mathrm{~mm}$; c) marcações das cargas hidráulicas.

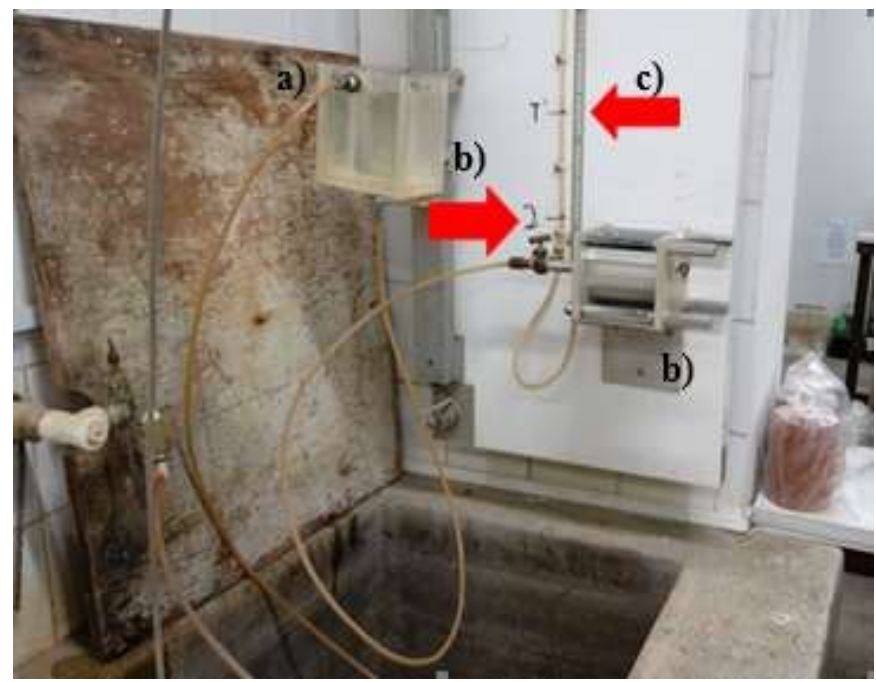

Fonte: Autores. 
Figura 7- Resultado do ensaio de pinhole test no solo.

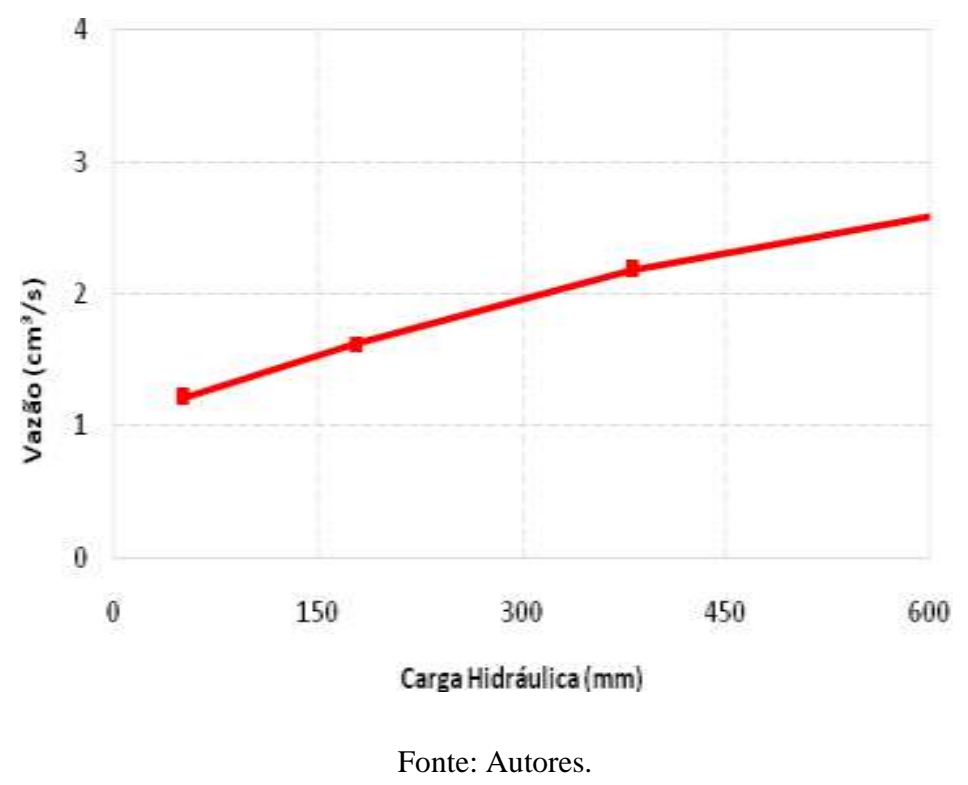

Observa-se que a carga hidráulica inicial de $25,4 \mathrm{~mm}$, atuante sobre a amostra de solo, gerou uma vazão média de $1,21 \mathrm{ml} / \mathrm{seg}$, apresentando uma coloração clara sem indícios de presença de partículas carreadas. Estas características foram mantidas nas cargas $180 \mathrm{~mm} 380 \mathrm{~mm}$ e $1020 \mathrm{~mm}$, tendo apresentado estas cargas como vazão média de: 1,62ml/s; 2,18ml/s e $3,37 \mathrm{ml} / \mathrm{s}$, respectivamente.

Durante a desmontagem do material, percebe-se que o furo permaneceu inalterado em todos os ensaios. A análise e interpretação dos resultados classificam o solo como sendo da classe ND1, ou seja, apresenta um comportamento não dispersivo.

\section{Ensaio Expansibilidade}

A Tabela 7 apresenta os resultados dos ensaios de expansibilidade. Observa-se que se comparado aos critérios propostos, o solo foi classificado como mau comportamento frente à erosão, enquanto que o RCD e as misturas como bom comportamento frente à erosão. A adição de RCD ao solo ocasionou uma redução de aproximadamente $85 \%$ na mistura S50R50 o que melhora o seu desempenho para uso em aterros sanitários.

Tabela 7- Resultado do ensaio de expansibilidade.

\begin{tabular}{llllll}
\hline Material & Solo & RCD & S90R10 & S70R30 & S50R50 \\
\hline $\boldsymbol{\varepsilon}(\%)$ & 14,57 & $2,07 \%$ & $8,83 \%$ & $2,30 \%$ & $2,13 \%$ \\
\hline
\end{tabular}

Fonte: Autores.

\section{Conclusão}

As camadas de coberturas dos aterros sanitários brasileiros são, em maior parte, feitas com solos compactados argilosos e tem a função de impedir a entrada de líquidos bem como evitar a saída de gases. Juntamente a isso, tem-se os resíduos de construção que são gerados na maioria das obras e depositados em locais irregulares. Ao combinar esses dois materiais percebe que é viável utilizar essa mistura para as camadas de coberturas. 
De acordo com os parâmetros geotécnicos exigidos pela USEPA (2004) e norma brasileira NBR 13896 (ABNT, 1997), a mistura S50R50 foi a que apresentou os melhores resultados.

Apesar de todas as misturas serem compatíveis com o uso como barreira hidráulica em aterros sanitários, no que diz respeito à condutividade hidráulica, o alto teor de finos fez com que as misturas S90R10 e S70R30 fossem classificadas como altamente plástica, de acordo com os limites de Atterberg. Esse alto teor de finos pode provocar, segundo (Moraci et al., 2017), fissuras por retração nos ciclos de secagem e umedecimento, além de uma dificuldade na compactação da camada.

Quanto à resistência por compressão simples, a adição de RCD a mistura S50R50, apresentou resultados satisfatório para o uso em camada de aterros sanitários, sendo cerca de 550\% superior ao valor de 0,20MPa proposto por (Daniel \& Wu, 1993).

Quanto à erodibilidade, os ensaios de crumb test e pinhole test apontaram o solo como material não erodível. Entretanto, o solo apresentou uma alta expansibilidade. A adição de RCD ao solo provocou uma diminuição da expansibilidade, sendo a mistura S50R50 a de melhores resultados.

Com a metodologia empregada, conclui-se que a melhor mistura foi a S50R50, pois é a mistura que mais consome $\mathrm{RCD}$, dando assim, uma destinação mais adequada a este material.

Os dados corroboram com pesquisas anteriores a de (Locastro \& Angelis, 2016; Rios, 2016; Costa, 2015; Dias, 2014), quanto ao uso do agregado reciclado em camada de cobertura de aterros sanitários, seja de forma parcial ou total, aprimorando o estado da arte sobre a temática.

Desta maneira, pode-se afirmar que a utilização do solo da encosta da Ilha de Itamaracá na Região Metropolitana do Recife-PE, pode ser utilizado com até 50\% de agregado reciclado.

A partir dos resultados encontrados, sugere-se algumas possibilidades para pesquisas futuras: analisar o comportamento de teores de agregados reciclados superiores a $50 \%$ quanto à condutividade hidráulica;analisar o comportamento da mistura S50R50, quanto ao aparecimento de fissuras, provocadas pelos ciclos de secagem e umedecimento; realizar ensaios de resistência à compressão simples com valores superiores a 50\% de RCD e analisar a incorporação de outros materiais, como fibras, para serem utilizados em sistema de camada de cobertura de aterros.

\section{Referências}

Alexandre, L. S., Silva, C. M., \& Castro, J. C. (2020). Predictive studies of solid waste production capacity in fast food restaurant susing the boot strap method and time series. International Journal of Advanced Engineering Researchand Science. 7.

Araujo, P. S. (2017) Análise do desempenho de um solo compactado utilizado na camada de cobertura de um aterro sanitário. Dissertação (Mestrado), Universidade Federal de Campina Grande Centro de Tecnologia e Recursos Naturais, Programa de Pós-Graduação em Engenharia Civil e Ambiental, Campina Grande.

Akhtar, A., \& Sarmah, A. K. (2018) Construction and demolition waste generation and properties of recycled aggregate concrete: A global perspective. Journal of Cleaner Production (Vol. 186, pp.262-281).

American Society For Testing And Materials (ASTM). (1990). Annual book of ASTM standards. Section 4: Construction, v.04: Soil and rock; dimension stone; geosynthetics. Philadelphia.

ASTM.D422-63. (2007) e2.Standard Test Method for Particle-Size Analysis of Soils (Withdrawn 2016). West Conshohocken, PA.

ASTM.D1557-12e1. (2012). Standard Test Methods for Laboratory Compaction Characteristics of Soil Using Modified Effort. West Conshohocken, PA.

ASTM.D6572-13e2. (2013a). Standard Test Methods for Determining Dispersive Characteristics of Clayey Soils by the Crumb Test. West Conshohocken, PA.

ASTM.D4647M-13. (2013b). Standard Test Methods for Identification and Classification of Dispersive Clay Soils by the Pinhole Test. West Conshohocken, PA.

ASTM.D854-14. (2014). Standard Test Methods for Specific Gravity of Soil Solids by Water Pycnometer. West Conshohocken, PA.

ASTM.D2166M-16 (2016). Standard Test Method for Unconfined Compressive Strength of Cohesive Soil. West Conshohocken, PA.

ASTM.D6913M-17. (2017a). Standard Test Methods for Particle-Size Distribution (Gradation) of Soils Using Sieve Analysis. West Conshohocken, PA, 2017. 
ASTM.D4318-17e1. (2017b). Standard Test Methods for Liquid Limit, Plastic Limit, and Plasticity Index of Soils. West Conshohocken, PA.

Associação Brasileira De Normas Técnicas- ABNT. (1997). NBR 13896: Aterros de resíduos não perigosos - Critérios para projeto, implantação e operação.

ABNT. (2000). NBR 14.545: Solo - Determinação do coeficiente de permeabilidade de solos argilosos a carga variável. Rio de Janeiro.

Carvalho, J. C., Gitirana Jr., G. F. N., Machado, S. L., Mascarenha, M. M. A., \& Silva filho, F. C. (2015). (Org.). Solos não saturados no contexto geotécnico. 1. ed. São Paulo: Associação Brasileira de Mecânica dos Solos e Engenharia Geotécnica, pp. 759.

Companhia de Tecnologia de Saneamento Ambiental - CETESB. (1993). Resíduos Sólidos Industriais. 2a ed. São Paulo, SP, 1993.

Costa, C. M. C. (2015) Avaliação da fissuração por ressecamento em camadas de cobertura de aterros sanitários utilizando materiais alternativos. Tese (Doutorado), Departamento de Engenharia Civil e Ambiental, Universidade de Brasília, Brasília.

Costa, C. M. C., Silva, T. J. L., Santos, J. R., Ribeiro, L. F. M., \& Gurjão, C. M. C. (2020). Avaliação do ressecamento da camada de cobertura utilizando solo com adição de fibras pet por meio de análise de imagens. Ciências Tecnológicas, Exatas e da Terra e seu Alto Grau de Aplicabilidade. Atena Editora, pp. 1331.

Daniel, D. E., \& Wu, Y. K. (1993). Compacted clay liners and covers for arid sites. Journal Geotechnical Engineering, ASCE (Vol. 119, pp.223-237).

Departamento Nacional De Estradas De Rodagem. (2002). DNER-ME 160/2002: Solos - Determinação da expansibilidade -Método de ensaio. Brasília.

Dias, M. C. C. (2014). Viabilidade do uso de solo tropical e resíduo de construção civil em sistemas de cobertura de aterro sanitário. Dissertação (Mestrado), Universidade Federal de Goiás, Escola de Engenharia civil Programa de Pós-Graduação em Geotecnica, Estruturas e Construção Civil, Goias.

Instituto Brasileiro de Geografia e Estatística (IBGE). (2018). Conheça Cidades e Estados do Brasil. Disponível em: <https://cidades.ibge.gov.br>..

Locastro, J. K, \& Angelis, B. L. D. (2016). Barreiras de impermeabilização: configurações aplicadas em aterros sanitários. Revista Eletrônica em Gestão, Educação e Tecnologia Ambiental, Santa Maria (Vol. 20, pp.200-210).

Macedo, M. C. (2016). Investigação sobre o comportamento geomecânico de misturas de solocom resíduos de pneus e resíduos de construção e demolição. Dissertação(Mestrado), Universidade de Pernambuco. Programa de Pós-Graduação em Engenharia Civil, Recife.

Manoharan, E., Othman, N., Mohammad, R., \& Tobi, S. U. M. (2020). Integrated Approach as Sustainable Environmental Technique for Managing Construction Waste: A review. Journal of Enviromental Treatment Tecniques, (Vol. 8, pp. 560-566).

Moraci, N., Busana, S., Cortellazzo, G., Favaretti, M., Mandaglio, M. C., \& Schepis, M. (2017). Design and construction of a compacted clay liner in cover system of a municipal solid waste (MSW) landfill using nonstandard procedures. Canadian Geotechnical Journal (Vol. 55, pp.1182-1192). http://dx.doi.org/10.1139/cgj-2017-0371.

Ortega, J. C. B., \& Campos, T. M. P. (2018) Caracterização fisica, mineralogia e morfologica de um solo residual compactado com problemas erosivos. Editora Atena, pp. 198.

Park, J, \& Tucker, R. (2016). Overcoming barriers to the reuse of construction waste material in Australia: a review of the literature. International Journal Of Construction Management, (Vol. 17, pp. 228-237).

Paz, D. H. F., Lafayette, K. P. V., Holanda, M. J. O., Sobral, M. C. M., \& Costa, L. A. R. C. (2020). Assessment of environmental impact risks arising from the illegal dumping of construction waste in Brazil. Environment, Development and Sustainability (Vol. 22, pp. 2289-2304). https://doi.org/10.1007/s10668018-0289-6

Pereira, A. S., Shitsuka, D. M., Parreira, F.. J. \& Shitsuka, R. (2018) Metodologia da pesquisa científica 1. ed. - Santa Maria, RS : UFSM, NTE.

Rios, D. C. (2016). Aplicação de dois modelos de balanço hídrico para estudo de camada de cobertura de aterro sanitário utilizando solo e resíduo da construção civil (RCC). Dissertação (Mestrado), Universidade Estadual de Feira de Santana, Programa de Pós-Graduação em Engenharia Civil e Ambiental, Bahia.

Santa, D. G., Cola, S., Tateo, F., \& Galgaro, A. (2020). Hydraulic conductivity changes in compacted clayey barriers due to temperature variations in landfill top covers. Bull EngGeol Environ (Vol. 79, pp. 2893-2905) https://doi.org/10.1007/s10064-020-01726-w

Scarin, P. B. R., \& Bernardes, G. P. (2016). Utilização do Índice de Perda ao Fogo para Caracterização de Perfil de Intemperismo de Solo Residual e de Horizontes de Deposição do Terciário de Taubaté. Anais do Congresso Brasileiro de Mecânica dos Solos e Engenharia Geotécnica, Belo Horizonte, MG, Brasil.

Silva, A. A. C., Fucale, S., \& Ferreiras. R. M. (2019). Efeito da adição de resíduos da construção e demolição (RCD) nas propriedades hidromecânicas de um solo areno-argiloso. Matéria (Rio de Janeiro) (Vol. 24, pp. 1-12).

Souza, L. N., \& Balduino, A. R. (2020). Reuse of waste from the manufacture of the granilite floor. International Journal of Advanced Engineering Research and Science. (Vol. 7).

USEPA (2004).Assessment and Recommendations for Improving the Performance of Waste Containment Systems. Washington, DC: EPA. < http://nepis.epa.gov/Adobe/PDF/P1001O83.pdf.>

Yazdanbakhsh, A. (2018). A bi-level environmental impact assessment framework for comparing construction and demolition waste management strategies. Waste Management (Vol. 77, pp.401-412,). http://dx.doi.org/10.1016/j.wasman.2018.04.024. 